\title{
Große Koalitionen in Deutschland und Österreich*
}

\author{
Kurzfassung
}

Große Koalitionen stellen einen demokratietheoretischen Sonderfall dar, der sich signifikant von einer „Kleinen Koalition“ unterscheiden kann: hinsichtlich der Strukturen, Ursachen und Auswirkungen. Diese Unterschiede werden in der Untersuchung unter Berücksichtigung der dafür einschlägigen Regierungskoalitionen in Deutschland und Österreich auf Bundesebene erfasst und diskutiert. Der Vergleich zwischen Deutschland und Österreich soll zeigen, welche unterschiedlichen Faktoren in Deutschland und Österreich zu Großen Koalitionen geführt haben, warum Große Koalitionen in Österreich wesentlich häufiger gebildet wurden und weshalb sie dort lange Zeit als Normal- bzw. Regelfall breite Akzeptanz fanden. Zudem soll der Vergleich zur Definition eines spezifischen Typs Großer Koalitionen (der „Monopolisierenden Großen Koalition") beitragen, der in beiden Ländern bei einer Gesamtbetrachtung - bislang - den dominierenden Typ darstellt, aber auch zeigen, welche Unterschiede neben den vielfältigen Gemeinsamkeiten zwischen den Auswirkungen Großer Koalitionen in Deutschland und Österreich existieren.

* Ich danke Eberhard Schuett-Wetschky sowie den beiden anonymen Gutachtern der ZPol für die äußerst wertvollen Hinweise zur ersten Fassung dieses Beitrags. 


\section{Inhalt}

1. Einleitung 6

2. Zur Einordnung und Definition Großer Koalitionen 8

3. Ursachen Großer Koalitionen 9

a) Österreich $\quad 12$

b) Bundesrepublik Deutschland 15

c) Zusammenfassung 17

4. Auswirkungen Großer Koalitionen 18

a) Repräsentation der Wähler 19

b) Stärke der Regierungsmehrheit 22

c) Dimensionen der Gewaltenteilung 23

(1) Horizontale Gewaltenteilung 24

(2) Bikamerale Gewaltenteilung 26

(3) Konstitutionelle Gewaltenteilung 29

(4) Temporale Gewaltenteilung 31

d) Zusammenfassung und Definition der Monopolisierenden

Großen Koalition 33

5. Fazit: Die Monopolisierende Große Koalition - Definition eines

Auslaufmodells?

\section{Einleitung}

Für die einen sind Große Koalitionen das „kleinste Übel“ (Pelinka 1987: 926), für die anderen ein „Widerspruch zum parlamentarischen System“ (Renzsch/Schieren 1997: 403) oder gar ,eine Art von demokratischem Sündenfall“ (Kielmansegg 2002: 9). Ob Große Koalitionen einen demokratischen Sündenfall darstellen, ist strittig. Unstrittig ist dagegen, dass sie einen demokratietheoretischen Sonderfall darstellen, der sich signifikant von einer „Kleinen Koalition“ unterscheiden kann: hinsichtlich der Strukturen, Ursachen und Auswirkungen. Diese werden im Folgenden unter Berücksichtigung der dafür einschlägigen Regierungskoalitionen in Deutschland und Österreich auf Bundesebene erfasst und diskutiert.

Im zweiten Abschnitt werden Große Koalitionen definiert. Da der Begriff seit geraumer Zeit im Sprachgebrauch ist, aber „keine analytische Kategorie der vergleichenden Politikwissenschaft" (Müller 2008: 499) bildet, ist es 
zunächst erforderlich, eine sinnvolle und praktikable Definition Großer Koalitionen zu erarbeiten.

Im dritten Abschnitt werden die Ursachen Großer Koalitionen erörtert die Große Koalition wird folglich als abhängige Variable untersucht. Da Große Koalitionen , den Erwartungen maßgeblicher Koalitionstheorien widersprechen“ (Müller 2008: 499), ist zu klären, welche Faktoren zur Bildung Großer Koalitionen führen können. Der Ländervergleich soll zeigen, welche unterschiedlichen Faktoren in Deutschland und Österreich zu Großen Koalitionen geführt haben, warum Große Koalitionen in Österreich wesentlich häufiger gebildet wurden und weshalb sie dort lange Zeit als Normal- bzw. Regelfall breite Akzeptanz fanden. Dabei ist darauf hinzuweisen, dass sich die Ursachen von Koalitionen - wie viele andere erklärende Variablen in der Politikwissenschaft - ,nicht objektiv im Sinne von eindeutig (zwingend und allgemeingültig) beweisen, sondern nur mehr oder minder plausibel begründen" (Schütt-Wetschky 1990: 51) lassen. Ob bzw. inwiefern theoretisch hergeleitete Variablen als kausale Mechanismen für die Koalitionsbildung fungieren (Müller 2008: 499 ff.), lässt sich folglich nur plausibel begründen, jedoch nicht endgültig beweisen.

Im vierten Abschnitt werden die wesentlichen spezifischen Auswirkungen Großer Koalitionen im Vergleich zu Kleinen Koalitionen sowie die damit verbundenen positiven und negativen Folgen herausgearbeitet - die Große Koalition wird folglich als unabhängige Variable untersucht. Der Ländervergleich soll zur Definition eines spezifischen Typs Großer Koalitionen (der „Monopolisierenden Großen Koalition“) beitragen, der in beiden Ländern bei einer Gesamtbetrachtung - bislang - den dominierenden Typ darstellt, aber auch zeigen, welche Unterschiede neben den vielfältigen Gemeinsamkeiten zwischen den Auswirkungen Großer Koalitionen in Deutschland und Österreich existieren. Die Darstellung der spezifischen Auswirkungen GroBer Koalitionen sowie der damit verbundenen möglichen Folgen geschieht weitgehend auf einer theoretisch-strukturellen Ebene. Folglich werden meist generelle Wirkungspotenziale, z. B. ein erhöhtes Steuerungs- bzw. Reformpotenzial, jedoch keine konkreten Wirkungen, wie eine erhöhte Reformtätigkeit, aufgezeigt. Diese ließen sich nur für jede Regierungskoalition (unter Berücksichtigung der Akteurkonstellation etc.) gesondert ermitteln.

Deutschland und Österreich wurden als Vergleichsländer ausgewählt, da sie mit Blick auf Große Koalitionen ,eine ziemliche Bandbreite abdecken“ (Müller 2008: 523) und bei einer Betrachtung der zentralen, für den Untersuchungsgegenstand relevanten Strukturmerkmale „most similar systems“ 
darstellen, was für das Untersuchungsdesign von zentraler Bedeutung ist. $\mathrm{Zu}$ diesen Strukturelementen zählen v. a. das Regierungssystem (die Art der Regierungsbildung sowie die gewaltenteilenden Strukturen), das Wahlsystem (die Verhältniswahl), das Rechtssystem (die Normenhierarchie), der Staatsaufbau (die föderale Struktur) bzw. das Kammersystem (Zweikammersystem) sowie das Parteiensystem (und die daraus resultierenden Strukturen Großer Koalitionen).

\section{Zur Einordnung und Definition Großer Koalitionen}

Große Koalitionen lassen sich nur schwer in die herkömmlichen analytischen Kategorien der vergleichenden Politikwissenschaft einordnen. Die relativ simple, aber letztlich unumgängliche Definition der Großen Koalition als Regierungsbündnis, an dem die beiden mandatsstärksten Parteien bzw. Fraktionen ${ }^{1}$ beteiligt sind, führt dazu, dass eine Vielzahl von Koalitionen mit höchst unterschiedlichen Formen und Größen unter diese Kategorie fallen können (Müller 2008: 500) - minimal winning coalitions (Minimale Mehrheitskoalitionen), minimum winning coalitions (Kleinstmögliche Mehrheitskoalitionen), undersized bzw. non-winning coalitions (Minderheitskoalitionen), oversized bzw. surplus coalitions (Überzählige Mehrheitskoalitionen) und damit auch all-party coalitions (Allparteienkoalitionen/Allparteienregierungen).

Um die Große Koalition zu einer sinnvollen Analysekategorie zu machen, ist es erforderlich, zwischen Großen Koalitionen als Minderheitskoalition, Minimaler Mehrheitskoalition und Überzähliger Mehrheitskoalition zu unterscheiden. Im ersten Fall bildet die Große Koalition ein Regierungsbündnis mit weniger als $50 \%$ der Parlamentsmandate, im zweiten Fall ein Regierungsbündnis mit mehr als $50 \%$ der Parlamentsmandate, bei dem der Austritt eines Partners aber zum Verlust der absoluten Mehrheit führt, und im dritten Fall ein Regierungsbündnis mit mehr als $50 \%$ der Parlamentsmandate, bei dem der Austritt eines Partners aber nicht zwingend zum Verlust der absoluten Mehrheit führt.

Die nachfolgenden Betrachtungen beschränken sich auf Große Koalitionen als Minimale Mehrheitskoalition. Dabei gilt: Minimale Mehrheitskoalitionen, die von den beiden mandatsstärksten Parteien gebildet werden, sind

1 Im Folgenden wird ausschließlich der Begriff der Partei verwendet. CDU und CSU werden dabei aufgrund der langjährigen Fraktionsgemeinschaft im Bund und der fehlenden Konkurrenz zwischen beiden Parteien - auf analytischer Ebene - als Einheit gewertet. 
als Große Koalition, ansonsten als Kleine Koalition zu werten. Die Große Koalition bildet damit wie die Kleine Koalition einen Subtyp der Minimalen Mehrheitskoalition, unter den allerdings erneut eine Vielzahl von Koalitionen mit höchst unterschiedlichen Formen und Größen fallen. Schließlich sagt die Tatsache, dass eine Große Koalition eine Minimale Mehrheitskoalition ist, noch wenig über deren Wesensgehalt bzw. Wirkungspotenzial aus. Aussagen darüber sind erst möglich, wenn man die Struktur des Parteiensystems als Kontextfaktor einbezieht.

Große Koalitionen können im Vergleich zu Kleinen Koalitionen nur dann einen spezifischen Wesensgehalt bzw. ein besonderes Wirkungspotenzial entfalten, wenn die Koalitionspartner (als Groß-, Haupt- bzw. Volksparteien) die beiden dominierenden Kräfte des Parteiensystems bilden und sich, u. a. mit einem Kandidaten für das Amt des Regierungschefs, als Hauptkonkurrenten um die Regierungsmacht gegenüberstehen - also unter den Bedingungen eines von zwei Parteien dominierten Mehrparteiensystems. Darunter fallen Parteiensysteme, bei denen die beiden größten Parteien in der Regel über mindestens zwei Drittel der Parlamentsmandate verfügen - nach der Parteiensystemeinteilung von Ware (1996: 162) etwa "two-and-a-half party systems“ sowie ,systems with two large parties“ - und damit (zumindest bis jetzt) die Parteiensysteme Deutschlands und Österreichs. Somit konzentrieren sich die nachfolgenden Betrachtungen auf Große Koalitionen als Minimale Mehrheitskoalition in einem von zwei Parteien dominierten Mehrparteiensystem. Welchen „Charakter" Große Koalitionen in dieser Form bzw. unter diesen Bedingungen entfalten können, wird bei der Darstellung der spezifischen Auswirkungen Großer Koalitionen deutlich.

\section{Ursachen Großer Koalitionen}

Klassische Koalitionstheorien basieren in der Regel auf der (impliziten) Annahme, dass Parteien - v. a. zur Sicherung der Regierungsstabilität und -funktionalität - bestrebt sind, „majority governments“ (Mehrheitsregierungen) zu bilden, also „minority“ bzw. „undersized governments“ (Minderheitsregierungen) zu vermeiden. Sie sagen folglich ,die Bildung regierungsfähiger, d. h. mehrheitsfähiger Koalitionen voraus" (Nolte 1988: 235). Zudem basieren sie auf der Annahme, Parteien seien Nutzen maximierende und rationale Akteure. Vor diesem Hintergrund wurden Parteien Motive zur Koalitionsbildung unterstellt, zunächst das office-seeking-Motiv: das Streben nach einer maximalen Anzahl an Regierungsämtern, das in der Theorie zu minimal winning 
coalitions bzw. - im Idealfall - minimum winning coalitions führt (Gamson 1961; Riker 1962). Mit dem office-seeking-Motiv allein konnte jedoch eine Vielzahl von Koalitionen nicht erklärt werden (Kropp 2008: 517). Es ist deshalb um das policy-seeking-Motiv ergänzt worden: das Streben nach der bestmöglichen Durchsetzung politischer Inhalte, das in der Theorie in Kombination mit dem office-seeking-Motiv zu ,minimal connected winning coalitions“ bzw. „minimal range coalitions“ (Axelrod 1970; De Swaan 1973) führt. Da die Erklärungs- bzw. Prognosefähigkeit von Koalitionstheorien dadurch allerdings nur begrenzt erhöht werden konnte (Nolte 1988: 231), mussten weitere Bestimmungsfaktoren des Koalitionsverhaltens gefunden werden, z. B. vote-seeking-Motive: das Streben nach einer maximalen Anzahl an Wählerstimmen (Strøm 1990), mit dessen Hilfe z. B. das Entstehen von Minderheitsregierungen relativ gut erklärt werden kann (Kropp 2008: 517 ff.). Doch trotz ständiger Modifizierungen und Erweiterungen der koalitionstheoretischen Annahmen, u. a. auch um die Faktoren „Bekanntheit“ und „Trägheit“, ist deren Erklärungs- bzw. Prognosekraft insgesamt begrenzt geblieben (Nolte 1988: 244 f.). Grundsätzlich ist festzuhalten, dass Koalitionen ,allein mit akteurstheoretischen Rational-Choice-Ansätzen und der Struktur von Parteiensystemen“ (Kropp u. a. 2002: 10) nicht erklärt werden können. So lässt sich auch die Bildung Großer Koalitionen - unter den Bedingungen eines von zwei Parteien dominierten Mehrparteiensystems - mit Nutzen maximierenden und rationalen Motiven nur schwer erklären.

In der hier untersuchten Form sind Große Koalitionen zwar minimal winning coalitions, sogar minimum number of parties coalitions (Minimale Mehrheitskoalitionen mit der kleinstmöglichen Zahl an Parteien), die aufgrund der verhältnismäßig niedrigen Transaktionskosten vorteilhaft erscheinen (Leiserson 1966), möglicherweise sogar minimal connected winning coalitions bzw. minimal range coalitions. Es gibt jedoch gewichtige vote-, policy- und v. a. office-seeking-Motive, die gegen die Bildung Großer Koalitionen sprechen. So haben die Parteien in einer Großen Koalition aufgrund ihrer spezifischen Stellung im Parteiensystem auf dem Wählermarkt relativ „viel zu verlieren und verhältnismäßig wenig zu gewinnen“" (Müller 2008: 507), aufgrund ihres spezifischen Konkurrenzverhältnisses im Regierungsprozess meist größere Probleme, ,sich mit dem Regierungspartner zu einigen“ (Müller 2008: 507), und aufgrund ihrer größeren Symmetrie im Vergleich zu Parteien in einer Kleinen Koalition, ein hohes Maß an Ämterverzicht“ (Müller 2008: 504) in Kauf zu nehmen. Da Große Koalitionen also entgegen 
gewichtiger Nutzen maximierender und rationaler Motive geschlossen werden, ist davon auszugehen, dass v. a. Zwänge zu Großen Koalitionen führen.

Ausgehend vom Parlament als Ort der Regierungskreation und Basis der Koalitionsformation werden im Folgenden exogene und endogene Zwänge - mit jeweils hoher und mittlerer Intensität - unterschieden, die separat oder in Kombination auftreten und zur Bildung Großer Koalitionen führen können. Zudem wird unterstellt, dass Parteien bestrebt sind, Mehrheitsregierungen zu bilden, also eine Große Koalition einer Minderheitsregierung grundsätzlich vorziehen. Diese Grundannahme trifft in jedem Fall auf Österreich und Deutschland zu, wo Minderheitsregierungen als unerwünschte Krisenerscheinung bzw. Sonder- oder Ausnahmefall in Übergangszeiten gelten.

Endogene Zwänge sind parlamentarische Faktoren (im engeren Sinne), die die Bildung einer Kleinen Koalition verhindern oder erschweren und zur Vermeidung einer Minderheitsregierung oder Regierbarkeitsproblemen die Bildung einer grundsätzlich ungewollten Großen Koalition notwendig oder sinnvoll erscheinen lassen. Dazu zählen eine fehlende stabile ${ }^{2}$ parlamentarische Mehrheit und/oder eine fehlende programmatische Möglichkeit zur Bildung einer Kleinen Koalition. Endogene Zwänge hoher Intensität entstehen, wenn grundsätzlich keine mehrheitsfähige Alternative zur Großen Koalition besteht. Dies ist dann der Fall, wenn Parteien, z. B. Antisystemparteien (Sartori 1993: 132 f.) bzw. extremistische Parteien (Jesse 2008: 9), für die Bildung einer Kleinen Koalition unabdingbar sind, aber von anderen Parteien als nicht koalitionsfähig eingestuft werden. Unter diesen Umständen reduzieren „Demarkationslinien für die Koalitionspolitik“ (Kropp 2008: 528) die arithmetischen Koalitionsmöglichkeiten auf eine einzige politische Koalitionsmöglichkeit: die Große Koalition. Endogene Zwänge mittlerer Intensität entstehen, wenn grundsätzlich mehrheitsfähige Alternativen zur Großen Koalition bestehen, deren Bildung aber aufgrund der (zu erwartenden) zu geringen Stabilität ${ }^{3}$ bzw. der zu großen programmatischen Spannbreite, die in einer Koalition wiederum zu einer geringen Stabilität führen kann, erschwert wird.

Exogene Zwänge sind außerparlamentarische Faktoren, die die Bildung einer Großen Koalition aufgrund ihrer „moralischen“ Stärke, die einem

2 Eine parlamentarische Mehrheit ist ,stabil“, wenn sie in der Lage ist, die Regierung ins Amt zu bringen, im Amt zu halten und in aller Regel deren einfachgesetzliche Regierungsvorhaben (in der ersten Parlamentskammer) umzusetzen.

3 Gründe für eine zu geringe Stabilität können z. B. eine zu große Anzahl an notwendigen Koalitionspartnern oder eine zu kleine Parlamentsmehrheit bzw. eine fehlende „working majority“ sein. 
Bündnis der beiden Hauptkonkurrenten im Parteiensystem zuwächst, und/ oder aufgrund ihrer parlamentarischen Stärke, die z. B. eine verfassungsändernde Mehrheit einschließt, notwendig oder sinnvoll erscheinen lassen unabhängig davon, ob eine Kleine Koalition gebildet werden könnte. Dazu zählen wesentliche politische und/oder gesellschaftliche Herausforderungen bzw. daraus ableitbare Ziele. Exogene Zwänge hoher Intensität entstehen bei systemgefährdenden Herausforderungen, wie z. B. besonderen Krisen- und Ausnahmesituationen, die ein nationales Gefahrenpotenzial implizieren (Knorr 1975: 17), oder einer äußerst starken gesellschaftlichen Fragmentierung, bei der der mehrheitsdemokratische Wettbewerb eine Gefahr für die Systemstabilität bedeutet. Exogene Zwänge mittlerer Intensität entstehen bei substanziellen, jedoch nicht systemgefährdenden Herausforderungen, wie z. B. notwendigen unpopulären Aufgaben bzw. größeren Reformen des politischen Systems, oder einer mittleren gesellschaftlichen Fragmentierung bzw. hohen gesellschaftlichen Heterogenität, die Strukturen einer Proporzdemokratie (Lehmbruch 1996: 2) sinnvoll erscheinen lässt. ${ }^{4}$

\section{a) Österreich}

In Österreich wurde nach der Nationalratswahl 1945 eine sogenannte „Konzentrationsregierung" (Pelinka 2002: 69) gebildet. Diese bestand zunächst aus allen im Parlament vertretenen Parteien (ÖVP, SPÖ und KPÖ) und nach 1947 aus den zwei großen Parteien (ÖVP und SPÖ), in beiden Fällen aus einer Großen Koalition als Überzählige Mehrheitskoalition, die nach der Nationalratswahl 1949 bis 1966 in der Form einer Großen Koalition als Minimale Mehrheitskoalition fortgesetzt wurde. Bis 1955 lässt sich diese erste Phase Großer Koalitionen primär auf einen exogenen Zwang hoher Intensität zurückführen: die Folgewirkungen des Zweiten Weltkriegs, d. h. die Besatzungszeit bzw. die eingeschränkte nationale Souveränität des Landes, die ohne Frage als Zeit der Systembedrohung gewertet werden kann. Dass die „ÖsterreichFrage" die Bildung Großer Koalitionen in dieser Phase primär verursacht hat, wird neben der Tatsache, dass sie als Bestandteil der Koalitionsstrategien vielfältig dokumentiert ist (Müller 2008: 515), u. a. dadurch deutlich, dass zwischen 1945 und 1949 trotz einer absoluten Mehrheit für die ÖVP keine Einparteiregierung, sondern eine Große Koalition als Überzählige

4 Fraglich ist dabei aus analytischer Sicht, ob eine Große Koalition aufgrund exogener Zwänge gegründet wurde oder nur mit diesen (im Nachhinein) begründet wurde. 
Mehrheitskoalition, zunächst sogar in der Form einer Allparteienregierung, gebildet wurde.

Die Lösung der „Österreich-Frage“ 1955 entzog zwar „der ,permanenten Koalition“ ihre wichtigste Begründung“" (Pelinka 2002: 75), doch wurde die Große Koalition bis 1966 fortgesetzt. Dies ist primär auf einen endogenen Zwang hoher Intensität zurückzuführen: die fehlende Koalitionsfähigkeit der FPÖ, die in diesem Zeitraum überwiegend das Monopol auf eine Koalitionsalternative besaß, sowie der KuL (Kommunisten und Linkssozialisten), mit der die ÖVP nach der Nationalratswahl 1956 eine (arithmetische) Mehrheit gehabt hätte. Schließlich war eine Koalition aus ÖVP und KuL ebenso wenig möglich wie eine Kleine Koalition unter Beteiligung der FPÖ, die das „,deutschnationale Lager“ bzw. - nach Pelinka (2002: 76) - , in ihrer Gesamtheit eine Partei in der Kontinuität der NSDAP“ repräsentierte und damit trotz gewisser Annäherungsversuche - für ÖVP und SPÖ nicht als Koalitionspartner infrage kam.

Zudem wurde die Fortsetzung der Großen Koalition durch einen exogenen Zwang mittlerer Intensität forciert: die überaus große Zustimmung zur Großen Koalition bzw. die ,politische Kultur der Konkordanzdemokratie“, die die nicht unerheblich fragmentierte österreichische Gesellschaft, die Lager(mentalität) mit ,intakten Feindbildern“ (Pelinka/Rosenberger 2003: 67 f.), „überwölbte“ und das Entstehen einer „zentrifugalen Demokratie“, wie sie sich in der ersten Republik herausgebildet hatte (Pelinka 1999: 15), „unterdrückte“. Das „konkordanzdemokratische Denken“ wurde auch dadurch begünstigt, dass bis 1998 in sieben und danach noch in fünf von neun Bundesländern Österreichs eine proportionale Regierungsbeteiligung aller im Landtag vertretenen Parteien verfassungsrechtlich festgelegt war (Kropp u. a. 2002: 20). Dass die Existenz gesellschaftlicher Lager bzw. die Kultur der Konkordanz die Bildung Großer Koalitionen in dieser ersten Phase nur zusätzlich forciert hat, wird $u$. a. dadurch deutlich, dass nach der Nationalratswahl 1966 eine lange Phase von Einparteiregierungen (in aller Regel Mehrheitsregierungen) gebildet wurde; dass ihr dennoch eine nicht zu vernachlässigende Bedeutung zukommt, zeigt sich darin, dass die nach 1966 gebildeten Einparteiregierungen eine relativ starke Konsensorientierung erkennen ließen. So war ihnen ,die parlamentarische Zustimmung der Opposition [...] wichtig genug [...], dieser bei den Politikinhalten Konzessionen zu machen“ (Müller 1988: 339).

Erst nach der Nationalratswahl 1983 wurde aufgrund einer fehlenden absoluten Parlamentsmehrheit für eine Partei wieder eine Koalition gebildet - 
allerdings keine Große Koalition. Nachdem sich die FPÖ 1970 durch die Duldung einer kurzzeitigen SPÖ-Minderheitsregierung und die in den 70erund 80er-Jahren eingeleitete Profilierung als liberale Partei an die SPÖ angenähert hatte, wurde erstmals eine Kleine Koalition aus SPÖ und FPÖ möglich. Die „Re-Positionierung“ (Müller 1988: 323) der FPÖ führte 1986 jedoch zum Auseinanderbrechen dieser Koalition sowie zu vorzeitigen Neuwahlen und der anschließenden Bildung einer Großen Koalition, die bis 2000 fortgesetzt wurde.

Diese zweite Phase Großer Koalitionen lässt sich primär auf einen endogenen Zwang hoher Intensität zurückführen: die fehlende Koalitionsfähigkeit der FPÖ, die in diesem Zeitraum wieder das Monopol auf eine Koalitionsalternative besaß. Schließlich verhinderte „der als ,rechtspopulistisch“ mit ,extremistischer Ausdrucksweise' eingeschätzte Kurs der FPÖ“ (Pelinka 2002: 79) die Bildung einer Kleinen Koalition. Dass das Profil der FPÖ die Bildung Großer Koalitionen in dieser Phase primär verursacht hat, wird u. a. dadurch deutlich, dass die 2000 gebildete FPÖ-ÖVP-Koalition ${ }^{5}$ erhebliche nationale und internationale Proteste hervorrief.

Die Bildung Großer Koalitionen wurde bis 1995 zudem durch einen exogenen Zwang mittlerer Intensität forciert: den EU-Beitritt Österreichs, der von der 1987 gebildeten Großen Koalition angestrebt wurde, und nur von ihr realisiert werden konnte (Müller 2008: $511 \mathrm{ff}$.). Dass dem angestrebten EUBeitritt eine nicht zu vernachlässigende Bedeutung zukommt, zeigt sich darin, dass die Große Koalition unmittelbar nach dessen Zustandekommen infrage gestellt wurde (Müller 2008: 511 f.); dass er die Bildung Großer Koalitionen in dieser Phase nur zusätzlich forciert hat, wird indessen u. a. dadurch deutlich, dass die Große Koalition dennoch bis 2000 fortgesetzt wurde.

Nach der langen Phase von Einparteiregierungen und der erstmaligen Bildung einer Kleinen Koalition dürfte der Kultur der Konkordanz bzw. der Existenz gesellschaftlicher Lager indessen kaum mehr eine nennenswerte Bedeutung beigemessen werden. Darauf deuten auch die starke Erosion der „Zweiten permanenten Großen Koalition“ und die Entwicklungen nach der Nationalratswahl 1999 hin. So lässt sich spätestens am Ende der zweiten Phase Großer Koalitionen eine „Auflösung der Subkulturen“ sowie ein „Ende der ,Lagermentalität"“ bzw. eine Abnahme der Fragmentierung der österreichischen Gesellschaft sowie eine Zunahme der Konfliktfähigkeit der

5 Die ÖVP stellte den Bundeskanzler, erhielt aber bei der Nationalratswahl 1999415 Stimmen weniger als die FPÖ, weshalb die Koalition nicht als ÖVP-FPÖ-Koalition gewertet wird. 
österreichischen Demokratie konstatieren (Pelinka/Rosenberger 2003: 177). Die Existenz gesellschaftlicher Lager bzw. die Kultur der Konkordanz vermag somit kaum zu erklären, warum in Österreich Große Koalitionen wesentlich häufiger gebildet wurden als in Deutschland, wohl aber, warum sie dort lange Zeit als Normal- bzw. Regelfall breite Akzeptanz fanden. Mit der Zunahme der Konfliktfähigkeit nahm diese Akzeptanz jedoch deutlich ab.

Nachdem die „zweite permanente Große Koalition“ in den 90er-Jahren drastisch an Zustimmung und Rückhalt verlor und die FPÖ deutlich an Wählerstimmen gewann sowie bei der Nationalratswahl 1999 die ÖVP als zweitstärkste Partei ablösen konnte, geriet diese in Zugzwang. Dabei entschied sie sich schließlich entgegen ihrer ursprünglichen Absicht, in die Opposition zu gehen, sowie nach gescheiterten Verhandlungen mit der SPÖ für eine Koalition mit der FPÖ. Dies rief zum Teil heftige Proteste hervor, führte jedoch nicht zu einer Destabilisierung des Systems - allerdings in gewisser Weise zu einer Destabilisierung der FPÖ. Schließlich spaltete sich diese 2005 in FPÖ und BZÖ (Bündnis Zukunft Österreich), das im Wesentlichen von der alten FPÖ-Spitze getragen wurde und die Koalition mit der ÖVP fortsetzte.

Letztlich mussten dadurch nach den Nationalratswahlen 2006 und 2008 erneut Große Koalitionen gebildet werden. Diese dritte Phase Großer Koalitionen lässt sich schließlich auf einen endogenen Zwang hoher bzw. mittlerer Intensität zurückführen: eine fehlende parlamentarische Mehrheit zur Bildung einer Kleinen Koalition aus zwei Parteien und eine fehlende stabile parlamentarische Mehrheit bzw. programmatische Möglichkeit zur Bildung einer Kleinen Koalition aus drei Parteien in dem entstandenen Fünf-Parteiensystem. In diesem wurde v. a. durch die FPÖ, aber auch durch das BZÖ das Set der arithmetischen Koalitionsmöglichkeiten erheblich reduziert und zumindest nach der Nationalratswahl 2006 nur die Große Koalition als mehrheitsfähige Alternative übrig gelassen.

\section{b) Bundesrepublik Deutschland}

In der Bundesrepublik Deutschland sind in den Nachkriegsjahren auf Länderebene in der Regel Koalitionen gebildet worden, die auf eine äußerst breite parlamentarische Mehrheit gestützt waren: Große Koalitionen bzw. Überzählige Mehrheitskoalitionen, häufig sogar unter Einbezug der KPD. Natürlich gab es auch Stimmen, die zur Konsenssicherung in der Nachkriegszeit bzw. zur Bewältigung aller ,mit der deutschen Frage zusammenhängenden Probleme“" (Knorr 1975: 17) nach 1949 - trotz der zum Teil 
höchst unterschiedlichen Positionen der großen Parteien - eine Große Koalition auf Bundesebene befürworteten. Diese konnten sich jedoch nicht (gegen Konrad Adenauer und Kurt Schumacher) durchsetzen (Wengst 1985: IX ff.). Schließlich wurde in Deutschland im Gegensatz zu Österreich nach dem Zweiten Weltkrieg auf Bundesebene lange Zeit keine Große Koalition gebildet - auch nicht nach dem Bau der Berliner Mauer bzw. der Bundestagswahl 1961, als die SPD eine Allparteienregierung forderte, oder der Spiegel-Affäre bzw. dem zeitweiligen Auseinanderbrechen CDU/CSU-FDP-Koalition 1962, als CDU/CSU und SPD erstmals Koalitionsverhandlungen führten (Kielmansegg 2007: 286 f.).

Erst nach der 1966 primär an haushalts- bzw. finanzpolitischen Streitfragen gescheiterten CDU/CSU-FDP-Koalition wurde nach einer vorübergehenden CDU/CSU-Minderheitsregierung eine Große Koalition auf Bundesebene gebildet. Diese lässt sich primär auf einen endogenen Zwang mittlerer Intensität zurückführen: eine trotz ernsthafter Koalitionsverhandlungen fehlende programmatische Möglichkeit zur Neuauflage einer Kleinen Koalition aus $\mathrm{CDU} / \mathrm{CSU}$ und $\mathrm{FDP}^{6}$ und eine trotz eines entsprechenden Koalitionsangebots der FDP an die SPD aus deren Sicht fehlende stabile parlamentarische Mehrheit zur Bildung einer SPD-FDP-Koalition. ${ }^{7}$ Zudem wurde die Bildung der Großen Koalition durch exogene Zwänge mittlerer Intensität forciert: die Rezession, ,in deren Schatten die rechtsradikale NPD erste Erfolge errang“ (Kielmansegg 2007: 288), bzw. das Motiv, die „haushalts- und finanzpolitischen Schwierigkeiten auf zwei annähernd gleichstarke Schultern zu verteilen und die unpopuläre Last gemeinsam zu tragen“ (Hildebrand 1984: 251), sowie die geplante Durchführung als notwendig erachteter struktureller Reformen, die nur durch ein Zusammenspiel von CDU/CSU und SPD möglich waren (Notstandsartikel, Neuordnung der Finanzen und Einführung der Mehrheitswahl). Dass die Rezession und die angestrebten Reformen die

6 Für die CDU/CSU und die FDP machte - neben strategischen Überlegungen der sich in einem Abwärtstrend befindenden FDP - v. a. ,die tiefe Zerrüttung ihrer geschiedenen Ehe eine Versöhnung nahezu aussichtslos“" (Hildebrand 1984: 244).

7 Mit einer Mehrheit von sechs Stimmen und einer in sich nicht geschlossenen FDP wäre ein Scheitern des (in diesem Fall notwendigen) konstruktiven Misstrauensvotums bzw. ein vorzeitiges Scheitern der Regierung nicht auszuschließen gewesen. Daher ist es nachvollziehbar, dass sich die SPD, die endlich ihre Regierungsfähigkeit beweisen - und nicht in kürzester Zeit widerlegen wollte - sowie eine gewisse Schlüsselstellung hatte, (vorerst) für eine Koalition mit der CDU/CSU entschied, für die die Große Koalition letztlich die einzige Alternative zum Gang in die Opposition bildete (Hildebrand 1984: 244 ff.). 
Bildung der ersten Großen Koalition nur zusätzlich forcierten, wird u. a. dadurch deutlich, dass 1966 in alle Richtungen mit offenem Ergebnis verhandelt wurde (Knorr 1975: 92 ff.); dass ihnen dennoch eine nicht zu vernachlässigende Bedeutung zukommt, zeigt sich darin, dass sie die Notwendigkeit „einer soliden und stabilen Regierungsbasis“ (Knorr 1975: 94) forcierten. Schließlich erhöhten die politischen Herausforderungen das Risiko des Scheiterns einer sozial-liberalen Koalition. So wurde erst 1969 eine sozial-liberale Koalition gebildet - welche eine lange Phase Kleiner Koalitionen einläutete, die nur für kurze Zeit durch eine SPD-Minderheitsregierung unterbrochen und 2005 durch die zweite Große Koalition beendet wurde.

Die zweite Große Koalition lässt sich auf einen endogenen Zwang mittlerer Intensität zurückführen: eine fehlende parlamentarische Mehrheit zur Bildung einer Kleinen Koalition aus zwei Parteien und eine fehlende stabile parlamentarische Mehrheit bzw. programmatische Möglichkeit zur Bildung einer Kleinen Koalition aus drei Parteien in dem neu entstandenen Fünf-Parteiensystem. In diesem wurde durch „Die Linke“ das Set der arithmetischen Koalitionsmöglichkeiten deutlich reduziert. Letztlich entstand eine ähnliche politische Machtkonstellation wie später bei den Nationalratswahl 2006 und 2008 in Österreich. Dass die beabsichtigte Föderalismusreform oder andere geplante Reformen, die der Zustimmung des Bundesrats und daher einer Mehrheit in der zweiten Kammer bedurften, keine exogenen Zwänge mittlerer Intensität darstellten bzw. die Bildung der zweiten Großen Koalition nicht forcierten, machen $\mathrm{u}$. a. der klare Lagerwahlkampf mit eindeutigen Koalitionsaussagen zugunsten Kleiner Koalitionen sowie die nach dem „Patt“ zwischen Rot-Grün und Schwarz-Gelb widerwillige Bildung der Großen Koalition deutlich (Gassert 2008: $103 \mathrm{f}$.).

\section{c) Zusammenfassung}

In Österreich gab es drei Phasen Großer Koalitionen auf Bundesebene. Die Bildung Großer Koalitionen in der ersten Phase (1945/1949-1966) ${ }^{8}$ wurde bis 1955 primär durch einen exogenen Zwang hoher Intensität, die „Österreich-Frage“, und danach primär durch einen endogenen Zwang hoher Intensität, die fehlende Koalitionsfähigkeit von FPÖ und KuL, verursacht sowie durch einen exogenen Zwang mittlerer Intensität, die Existenz gesellschaftlicher Lager bzw. die Kultur der Konkordanz, forciert. Die Bildung Großer Koalitionen in der zweiten Phase (1987-2000) wurde primär durch einen

8 Bis 1949 waren die Großen Koalitionen Überzählige Mehrheitskoalitionen. 
endogenen Zwang hoher Intensität, die fehlende Koalitionsfähigkeit der FPÖ, verursacht sowie bis 1995 durch einen exogenen Zwang mittlerer Intensität, den angestrebten EU-Beitritt, forciert. Die dritte Phase (seit 2007) wurde durch einen endogenen Zwang hoher bzw. mittlerer Intensität verursacht: das Fünf-Parteiensystem mit FPÖ und BZÖ als (zum Teil) nicht koalitionsfähige Parteien.

In der Bundesrepublik Deutschland gab es bisher nur zwei Große Koalitionen auf Bundesebene. Die erste Große Koalition (1966-1969) wurde primär durch einen endogenen Zwang mittlerer Intensität, eine fehlende programmatische Möglichkeit zur Bildung einer CDU/CSU-FDP-Koalition und eine fehlende stabile parlamentarische Mehrheit zur Bildung einer SPDFDP-Koalition, verursacht sowie durch exogene Zwänge mittlerer Intensität, die Rezession und angestrebte Reformen, forciert. Die zweite Große Koalition (seit 2005) wurde durch einen endogenen Zwang mittlerer Intensität verursacht: das Fünf-Parteiensystem mit der „Linken“ als nicht koalitionsfähige Partei.

Dass in Österreich wesentlich häufiger Große Koalitionen gebildet wurden als in der Bundesrepublik Deutschland, lässt sich auf zwei Gründe zurückführen: Zum einen führten die Folgewirkungen des Zweiten Weltkriegs in Deutschland nur auf Länderebene zu Großen Koalitionen. Zum anderen wurde das Set der arithmetischen Koalitionsmöglichkeiten in Österreich weitaus häufiger auf ein Set der politischen Koalitionsmöglichkeiten reduziert, das nur noch die Große Koalition als mehrheitsfähige Alternative übrig ließ. Die Existenz gesellschaftlicher Lager bzw. die Kultur der Konkordanz vermag indessen kaum zu erklären, warum in Österreich Große Koalitionen wesentlich häufiger gebildet wurden als in Deutschland, wohl aber, warum sie dort lange Zeit als Normal- bzw. Regelfall breite Akzeptanz fanden.

\section{Auswirkungen Großer Koalitionen}

Große Koalitionen sind - wie es Kurt Georg Kiesinger (1971: 185) ausgedrückt hat - „ein Ereignis, an das sich viele Hoffnungen und Sorgen [...] knüpfen“. Diese beziehen sich in erster Linie auf die spezifischen Auswirkungen Großer Koalitionen bzw. die konkreten Unterschiede zwischen kleinen und Großen Koalitionen, die sich im Wesentlichen in drei Bereichen zeigen: der Repräsentation der Wähler, der Stärke der Regierungsmehrheit und verschiedenen Dimensionen der Gewaltenteilung. 


\section{a) Repräsentation der Wähler}

Ein wesentlicher Unterschied zwischen kleinen und Großen Koalitionen zeigt sich im Anteil der Wählerstimmen der Regierungsparteien und damit in der Repräsentation der Wähler auf der parlamentarischen Entscheidungsebene bzw. der Regierungsebene. Die Unterscheidung zwischen der parlamentarischen Repräsentationsebene, dem Gesamtparlament, und der parlamentarischen Entscheidungsebene bzw. der Regierungsebene, der Regierungsmehrheit, trägt der häufig nicht hinreichend reflektierten Tatsache Rechnung, „dass eben diese Mehrheit und nicht das Gesamtparlament handelt" (SchuettWetschky 2005: 504). Folglich wird zwischen der formalen Beschlussfassung durch das gesamte Parlament und der - machtpolitisch weitaus bedeutsam und deshalb in diesem Beitrag vordergründig betrachteten - materiellen Entscheidung durch die Regierungsmehrheit unterschieden (Schuett-Wetschky 2005: 491 f.). Eine vergleichende Betrachtung Großer und Kleiner Koalitionen in beiden Ländern zeigt, dass der Repräsentationsgrad der Wähler auf der parlamentarischen Entscheidungsebene bzw. der Regierungsebene bei Großen Koalitionen höher ist als bei Kleinen Koalitionen (Tabelle 1 und 2).

Kleine Koalitionen stützen sich häufig auf eine absolute, zum Teil aber auch nur auf eine relative Mehrheit der Wählerstimmen. In Deutschland vereinten die Kleinen Koalitionen zwischen 46,9 und 58,2 \%9, in Österreich zwischen 52,3 und 53,8 \% der Wählerstimmen.

Große Koalitionen können sich hingegen in aller Regel auf eine qualifizierte Mehrheit der Wählerstimmen stützen. In Deutschland vereinten die Großen Koalitionen 86,9\% bzw. 69,4 \%, in Österreich zwischen 55,2 und 89,4 \% der Wählerstimmen.

Der Ländervergleich zeigt, dass in beiden Ländern der Anteil der Wählerstimmen Großer Koalitionen zum Teil wesentlich größer war als bei Kleinen Koalitionen, jedoch im Laufe der Zeit stark abgenommen hat. Deutlicher als in Deutschland zeigt sich diese Erosion - bislang - in Österreich, wo insgesamt drei Große Koalitionen (die Regierungskoalitionen nach den Nationalratswahlen 1994, 1995 und 2008) über weniger als zwei Drittel der Wählerstimmen verfügten - die jüngste sogar nur noch über 55,2\%.

9 Dabei fällt auf, dass - aufgrund der konzentrierenden Wirkung der Sperrklausel - sogar fünf Kleine Koalitionen über weniger als 50 \% der Wählerstimmen verfügten. 
Tabelle 1

Politische Mehrheitsverhältnisse in Deutschland

\begin{tabular}{|l|c|l|c|c|c|c|r|}
\hline Wahl & $R W$ & Koalition & Typ & $W S-R$ & $P M-R$ & $P M-O$ & $\begin{array}{r}P M- \\
G O\end{array}$ \\
\hline 14.08 .1949 & - & CDU/CSU-FDP-DP & KK & 46,9 & 51,7 & 48,3 & 32,6 \\
\hline 06.09 .1953 & UR & CDU/CSU-FDP-BHE-DP & ÜK & 63,9 & 68,6 & 31,4 & 31,0 \\
$(a b 1955)$ & UR & CDU/CSU-FDP-DP & ÜK & 58,0 & 63,1 & 36,9 & 31,0 \\
$(a b 1956)$ & UR & CDU/CSU-DP & UKK & 48,5 & 53,2 & 46,8 & 31,0 \\
\hline 15.09 .1957 & - & CDU/CSU-DP & ÜK & 53,6 & 57,7 & 42,3 & 34,0 \\
$(a b 1960)$ & - & CDU/CSU & EM & 53,6 & 57,7 & 42,3 & 34,0 \\
\hline 17.09 .1961 & UR & CDU/CSU-FDP & KK & 58,2 & 61,9 & 38,1 & 38,1 \\
\hline 19.09 .1965 & - & CDU/CSU-FDP & KK & 57,1 & 59,3 & 40,7 & 40,7 \\
$(a b 1966)$ & UR & CDU/CSU-SPD & GK & 86,9 & 90,1 & 9,9 & 9,9 \\
\hline 28.09 .1969 & UR & SPD-FDP & KK & 48,5 & 51,2 & 48,8 & 48,8 \\
\hline 19.11 .1972 & - & SPD-FDP & KK & 54,2 & 54,6 & 45,4 & 45,4 \\
\hline 03.10 .1976 & - & SPD-FDP & KK & 50,5 & 51,0 & 49,0 & 49,0 \\
\hline 05.10 .1980 & - & SPD-FDP & KK & 53,5 & 54,5 & 45,5 & 45,5 \\
$(a b 1982)$ & UR & CDU/CSU-FDP & KK & 55,1 & 56,1 & 43,9 & 43,9 \\
\hline 06.03 .1983 & - & CDU/CSU-FDP & KK & 55,8 & 55,8 & 44,2 & 38,8 \\
\hline 25.01 .1987 & - & CDU/CSU-FDP & KK & 53,4 & 54,1 & 45,9 & 37,4 \\
\hline 02.12 .1990 & - & CDU/CSU-FDP & KK & 54,8 & 60,1 & 39,9 & 36,1 \\
\hline 16.10 .1994 & - & CDU/CSU-FDP & KK & 48,4 & 50,7 & 49,3 & 37,5 \\
\hline 27.09 .1998 & VR & SPD-B'90/Grüne & KK & 47,6 & 51,6 & 48,4 & 36,6 \\
\hline 22.09 .2002 & - & SPD-B'90/Grüne & KK & 47,1 & 50,7 & 49,3 & 41,1 \\
\hline 18.09 .2005 & UR & CDU/CSU-SPD & GK & 69,4 & 73,0 & 27,0 & 9,9 \\
\hline
\end{tabular}

$\mathrm{RW}=$ Regierungswechsel, UR = unvollständiger Regierungswechsel (inkl. kleinerer Veränderungen bzw. Koalitionswechsel), VR = vollständiger Regierungswechsel, ÜK = Überzählige Mehrheitskoalition, GK = Große Koalition (als Minimale Mehrheitskoalition), $\mathrm{KK}=$ Kleine Koalition, EM = Einpartei-Mehrheitsregierung, MR = Minderheitsregierung, WS-R = Wählerstimmen der Regierungspartei(en), PM-R = Parlamentsmandate der Regierungspartei(en), PM-O = Parlamentsmandate der Oppositionspartei(en), PM$\mathrm{GO}=$ Parlamentsmandate der größten Oppositionspartei. Die Daten beziehen sich auf den Stand bei der Wahl. Kleinere Veränderungen (in Übergangszeiten) sowie die Sitze der West-Berliner Abgeordneten wurden nicht berücksichtigt. Quelle: Eigene Darstellung (Basis: Informationen des Bundestags). 
Tabelle 2

Politische Mehrheitsverhältnisse in Österreich

\begin{tabular}{|l|c|l|c|c|c|c|c|}
\hline Wahl & $R W$ & Koalition & ypp & $W S-R$ & $P M-R$ & $P M-O$ & $P M-G O$ \\
\hline 25.11 .1945 & - & ÖVP-SPÖ-KPÖ & ÜK & 99,8 & 100 & 0 & 0 \\
$(a b 1947)$ & UR & ÖVP-SPÖ & ÜK & 94,4 & 97,6 & 2,4 & 2,4 \\
\hline 09.10 .1949 & - & ÖVP-SPÖ & GK & 82,7 & 87,3 & 12,7 & 9,7 \\
\hline 22.02 .1953 & - & ÖVP-SPÖ & GK & 83,4 & 89,1 & 10,9 & 8,5 \\
\hline 13.05 .1956 & - & ÖVP-SPÖ & GK & 89,0 & 94,5 & 5,5 & 3,6 \\
\hline 10.05 .1959 & - & ÖVP-SPÖ & GK & 89,0 & 95,2 & 4,8 & 4,8 \\
\hline 18.11 .1962 & - & ÖVP-SPÖ & GK & 89,4 & 95,2 & 4,8 & 4,8 \\
\hline 06.03 .1966 & UR & ÖVP & EM & 48,4 & 51,5 & 48,5 & 44,8 \\
\hline 01.03 .1970 & VR & SPÖ & MR & 48,4 & 49,1 & 50,9 & 47,3 \\
\hline 10.10 .1971 & - & SPÖ & EM & 50,0 & 50,8 & 49,2 & 43,7 \\
\hline 05.10 .1975 & - & SPÖ & EM & 50,4 & 50,8 & 49,2 & 43,7 \\
\hline 06.05 .1979 & - & SPÖ & EM & 51,0 & 51,9 & 48,1 & 42,1 \\
\hline 24.04 .1983 & UR & SPÖ-FPÖ & KK & 52,6 & 55,7 & 44,3 & 44,3 \\
\hline 23.11 .1986 & UR & SPÖ-ÖVP & GK & 84,4 & 85,8 & 14,2 & 9,8 \\
\hline 07.10 .1990 & - & SPÖ-ÖVP & GK & 74,9 & 76,5 & 23,5 & 18,0 \\
\hline 09.10 .1994 & - & SPÖ-ÖVP & GK & 62,6 & 63,9 & 36,1 & 23,0 \\
\hline 17.12 .1995 & - & SPÖ-ÖVP & GK & 66,4 & 67,2 & 32,8 & 22,4 \\
\hline 03.10 .1999 & UR & FPÖ-ÖVP & KK & 53,8 & 56,8 & 43,2 & 35,5 \\
\hline 24.11 .2002 & - & ÖVP-FPÖ & KK & 52,3 & 53,0 & 47,0 & 37,7 \\
& & (ab 2005 BZÖ) & & & & & \\
\hline 01.10 .2006 & UR & SPÖ-ÖVP & GK & 69,7 & 73,2 & 26,8 & 11,5 \\
\hline 28.09 .2008 & - & SPÖ-ÖVP & GK & 55,2 & 59,0 & 41,0 & 18,6 \\
\hline
\end{tabular}

Abkürzungen siehe Tabelle 1. Quelle: Eigene Darstellung (Basis: Informationen des Nationalrats).

Der vergleichsweise hohe Repräsentationsgrad der Wähler auf der parlamentarischen Entscheidungsebene bzw. der Regierungsebene bei Großen Koalitionen ist grundsätzlich positiv zu bewerten. Schließlich können sich parlamentarische Entscheidungen auf einen breiten parlamentarischen Konsens ${ }^{10}$ bzw. eine breite Regierungsmehrheit und sich diese auf eine breite

10 Darin lässt sich aus einer bestimmten Perspektive durchaus ein Idealbild der Demokratie sehen (Knorr 1975: 20). 
Mehrheit der Wähler stützen ${ }^{11}$ - allerdings nur bei einer formal-arithmetischen, nicht zwingend bei einer materiell-qualitativen Betrachtung.

Es ist keineswegs davon auszugehen, dass die Wähler, die für die Parteien der Großen Koalition votierten, auch eine Große Koalition und deren Politik wünschten. So haben z. B. in Deutschland bei der Bundestagswahl 2005 insgesamt 69,4 \% für die Parteien der Großen Koalition gestimmt, doch nur $33 \%$ eine Große Koalition gewollt (Infratest dimap 2005). Mit deren Politik waren nach rund einem Jahr Amtszeit sogar nur $19 \%$ (43 \% der CDU/CSUWähler und $20 \%$ der SPD-Wähler) zufrieden (Infratest dimap 2006). Dies ist u. a. damit zu erklären, dass Große Koalitionen in nahezu allen Fällen ,große" Kompromisse schließen müssen und die Koalitionspartner dabei an Kontur einbüßen. Eine mögliche negative Folge könnte deshalb darin bestehen, dass zwar ein Großteil der Wähler von den Parteien der Großen Koalition repräsentiert wird, sich aber eine Vielzahl dieser Wähler nicht von der Großen Koalition repräsentiert fühlt.

\section{b) Stärke der Regierungsmehrheit}

Ein weiterer wesentlicher Unterschied zwischen kleinen und Großen Koalitionen besteht im Anteil der Parlamentsmandate der Regierungsparteien und damit in der Stärke der Regierungsmehrheit im Parlament. Eine vergleichende Betrachtung Großer und Kleiner Koalitionen in beiden Ländern zeigt, dass die Stärke der Regierungsmehrheit im Parlament bei Großen Koalitionen umfangreicher ist als bei Kleinen Koalitionen (Tabelle 1 und 2).

Kleine Koalitionen stützen sich nur auf eine - zum Teil äußerst knappe absolute Mehrheit im Parlament. In Deutschland vereinten die Kleinen Koalitionen zwischen 50,7 und 61,9\%, in Österreich zwischen 53,0 und 56,8\% der Parlamentsmandate.

Große Koalitionen können sich hingegen in aller Regel auf eine qualifizierte Mehrheit im Parlament stützen. In Deutschland vereinten die Großen Koalitionen 90,1 \% bzw. 73,0 \%, in Österreich zwischen 59,0 und 95,2 \% der Parlamentsmandate.

Der Ländervergleich zeigt, dass in beiden Ländern der Anteil der Parlamentsmandate analog zum Anteil der Wählerstimmen Großer Koalitionen zum Teil wesentlich größer war als bei Kleinen Koalitionen, jedoch im Laufe

11 Zumindest garantierten die Großen Koalitionen - bislang - und nicht, wie häufig behauptet, die Mechanismen der Verhältniswahl, dass sich Regierungen auf eine absolute Mehrheit der Wähler stützen. 
der Zeit stark abgenommen hat - v. a. in Österreich, wo insgesamt zwei Große Koalitionen (die Regierungskoalitionen nach den Nationalratswahlen 1994 und 2008) über weniger als zwei Drittel der Parlamentsmandate verfügten. Folglich wurde nur bei der Großen Koalition nach der Nationalratswahl 1995 die fehlende Zweidrittelmehrheit der Wählerstimmen durch die konzentrierenden Mechanismen des Wahlsystems in eine Zweidrittelmehrheit der Parlamentsmandate umgewandelt.

Die vergleichsweise umfangreiche Stärke der Regierungsmehrheit im Parlament bei Großen Koalitionen ist grundsätzlich positiv zu bewerten. Schließlich erhöht sich dadurch in gewisser Weise - die generelle „Koalitionstreue" der Regierungsparteien vorausgesetzt - die Stabilität der Regierung. ${ }^{12}$ Immerhin ist es de facto unmöglich, dass diese, z. B. aufgrund einiger „Abweichler“ oder „Überläufer“, ihre Mehrheit temporär oder dauerhaft verliert. Temporäre Regierbarkeitsprobleme durch „Abweichler“ in der Regierungsmehrheit, wie bei der rot-grünen Regierung 2001 in Deutschland vor der Entscheidung über den Afghanistan-Einsatz der Bundeswehr, oder dauerhafte Regierbarkeitsprobleme durch ein „Abschmelzen“ der Regierungsmehrheit, wie bei der sozial-liberalen Regierung 1972 in Deutschland, sind bei Großen Koalitionen kaum möglich.

Als negative Folge lässt sich indessen die „Exekutivlastigkeit“ des Parlaments, die sich aus der „komfortablen“ parlamentarischen Mehrheit in Verbindung mit der Oligarchisierung der Regierungsparteien ergibt, bzw. der daraus resultierende Bedeutungsverlust des einzelnen Abgeordneten sehen. Schließlich kann eine Regierung, die über eine überaus große bzw. qualifizierte Mehrheit der Parlamentsmandate verfügt, für Beschlüsse aber nur eine relative bzw. absolute Mehrheit der Parlamentsmandate benötigt, problemlos auf einige „Abweichler“ in den eigenen Reihen bzw. deren Meinung verzichten. So konnten in Deutschland Kurt Georg Kiesinger und Angela Merkel auch bei der Kanzlerwahl getrost auf 107 bzw. 51 Stimmen ,ihrer“ Regierungsmehrheit verzichten.

\section{c) Dimensionen der Gewaltenteilung}

Weitere wesentliche Unterschiede zwischen Kleinen und Großen Koalitionen zeigen sich in der horizontalen, bikameralen, konstitutionellen und temporalen

12 Die Stabilität der Regierung hängt natürlich nicht nur, aber doch maßgeblich von der Stärke der Regierungsmehrheit im Parlament ab. 
Gewaltenteilung - also denjenigen Dimensionen der Gewaltenteilung, die von den Kräfteverhältnissen im Parlament entscheidend beeinflusst werden.

\section{(1) Horizontale Gewaltenteilung}

Die horizontale Gewaltenteilung (Steffani 1979: 20) beschreibt grundsätzlich die Aufteilung der Staatsgewalt auf mehrere Staatsorgane einer föderalen Ebene - u. a. auf Parlament und Regierung. Entscheidend ist, dass dieses „klassische Gewaltenteilungskonzept“ in parlamentarischen Regierungssystemen nur in formaler Hinsicht existiert. In materieller Hinsicht werden Gesetzgebung und Vollziehung (politische Entscheidung) von der Regierungsmehrheit, der,,Aktionsgemeinschaft" von Regierung und Parlamentsmehrheit, und die Kontrolle (politische Opposition) von der Parlamentsminderheit wahrgenommen (Schuett-Wetschky 2001: 83 ff.). Die horizontale Gewaltenteilung steht also in parlamentarischen Regierungssystemen in materieller Hinsicht $u$. a. für eine parlamentarische Kontrolle der Regierung(smehrheit) durch die Parlamentsminderheit. Das bedeutet, dass die horizontale Gewaltenteilung faktisch von einer effektiven Kontrolle der Regierungsmehrheit und diese „von der Existenz einer funktionsfähigen Opposition abhängt“ (Knorr 1975: 242). Eine vergleichende Betrachtung Großer und Kleiner Koalitionen in beiden Ländern zeigt, dass die horizontale Gewaltenteilung bei Großen Koalitionen im Gegensatz zu Kleinen Koalitionen in aller Regel faktisch nur eingeschränkt gegeben ist (Tabelle 1 und 2).

Bei Kleinen Koalitionen ist die parlamentarische Kontrolle der Regierung(smehrheit) durch die Parlamentsminderheit in aller Regel uneingeschränkt gegeben, da meist alle parlamentarischen Kontrollinstrumente von der Opposition bzw. der größten Oppositionspartei allein genutzt werden können. So lag der Anteil der Parlamentsminderheit sowie in aller Regel auch der Anteil der größten Oppositionspartei ${ }^{13}$ bei Kleinen Koalitionen in Deutschland und Österreich nicht unter einem Drittel der Parlamentsmandate - der in beiden Ländern höchsten „Hürde“ zur Nutzung der parlamentarischen Kontrollinstrumente der Parlamentsminderheit.

Bei Großen Koalitionen ist die parlamentarische Kontrolle der Regierung(smehrheit) durch die Parlamentsminderheit in aller Regel nur

13 Nur in Deutschland lag der Anteil der größten Oppositionspartei in der ersten Wahlperiode - sowie auch in der zweiten Wahlperiode, in der die Regierung allerdings von Überzähligen Mehrheitskoalitionen gebildet wurde - unter einem Drittel der Parlamentsmandate. Dies ist auf die anfängliche Zersplitterung des Parteiensystems zurückzuführen. 
eingeschränkt gegeben, da meist nicht alle parlamentarischen Kontrollinstrumente von der Opposition bzw. der größten Oppositionspartei allein genutzt werden können ${ }^{14}$. So lag bei der ersten Großen Koalition in Deutschland der Anteil der Parlamentsminderheit bzw. der einzigen Oppositionspartei bei 9,9 \% der Parlamentsmandate. Damit war diese nicht in der Lage, eine Sondersitzung des Bundestags oder eine abstrakte Normenkontrolle zu verlangen (wofür ein Drittel der Mitglieder des Bundestags erforderlich ist; Art. 39 Abs. 3 Satz 3; Art. 93 Abs. 1 Nr. 2 GG) sowie einen Untersuchungsausschuss einzusetzen (wofür ein Viertel der Mitglieder des Bundestags erforderlich ist; Art. 44 Abs. 1 Satz 1 GG). Bei der zweiten Großen Koalition liegt der Anteil der Parlamentsminderheit bei 27,0 \% und der Anteil der größten Oppositionspartei bei 9,9 \% der Parlamentsmandate. Damit können die Oppositionsparteien keine Sondersitzung des Bundestags oder eine abstrakte Normenkontrolle verlangen und nur vereint einen Untersuchungsausschuss einsetzen. In Österreich lag bei den Großen Koalitionen der Anteil der Parlamentsminderheit mit zwei Ausnahmen (den Regierungskoalitionen nach den Nationalratswahlen 1994 und 2008) nicht über einem Drittel und der Anteil der größten Oppositionspartei nie über einem Viertel der Parlamentsmandate. Damit war die Opposition bei den meisten Großen Koalitionen nicht in der Lage, eine außerordentliche Tagung des Nationalrats oder eine abstrakte Normenkontrolle zu verlangen (wofür ein Drittel der Mitglieder des Nationalrats erforderlich ist; Art. 28 Abs. 2 Satz 2; Art. 140 Abs. 1 Satz 2 B-VG). ${ }^{15}$

Bei dem Ländervergleich fällt auf, dass in Österreich ein Drittel der Mitglieder des Nationalrats (oder des Bundesrats) - damit allerdings in der Regel nicht die Opposition zu einer Großen Koalition - auch eine Volksabstimmung bei einer Teiländerung der Bundesverfassung verlangen kann, jedoch nicht in der Lage ist, einen Untersuchungsausschuss einzusetzen (Art. 44 Abs. 3 BVG; $\S 33$ Abs. 1 GOG-NR), da dies im österreichischen Nationalrat Mehrheitsrecht ist - und als solches „zum toten Recht" (Pelinka/Rosenberger 2003: 124) wird.

Die faktische Einschränkung der horizontalen Gewaltenteilung bei Großen Koalitionen ist grundsätzlich negativ zu bewerten. Schließlich besteht durch die zum Teil fehlende Kontrolle der Regierung(smehrheit) die erhöhte Gefahr, dass die Regierungsmacht unbemerkt bzw. ungeahndet missbraucht

14 Die Opposition zu einer Großen Koalition hat aufgrund ihrer quantitativen Stärke allerdings nicht nur institutionelle, sondern auch personelle und inhaltliche Probleme, also Kapazitätsprobleme, ihrer Kontrollfunktion gerecht zu werden (Knorr 1975: 243).

15 Die oben genannte Möglichkeit zur Beantragung einer abstrakten Normenkontrolle besteht allerdings erst seit 1975 . 
wird. Auch wenn das Verhältnis zwischen Regierung und Regierungsfraktionen bei Großen Koalitionen zum Teil distanzierter sein mag als bei Kleinen Koalitionen, ist keineswegs davon auszugehen, dass die mangelnde oppositionelle Kontrolle durch eine regierungsinterne Kontrolle (Bereichsopposition etc.) kompensiert und damit sogar zum klassischen Konzept der Gewaltenteilung zurückgekehrt werden könnte (Knorr 1975: 24 ff.).

Als positive Folge lässt sich allenfalls das erhöhte Steuerungs- bzw. Reformpotenzial sehen, das sich im Bereich der Gesetzgebung durch die massive Einschränkung der Möglichkeit zur Normenkontrolle seitens des Verfassungsgerichts ergibt. Schließlich fällt dieses als Vetospieler zu den von den Regierungsparteien im Parlament beschlossenen Gesetzen weitestgehend aus, wenn die Opposition nicht in der Lage ist, ein abstraktes Normenkontrollverfahren zu initiieren - das in bestimmten Fällen durchaus zu einem nicht unerheblichen oppositionellen „Blockadeinstrument“ avancieren kann.

\section{(2) Bikamerale Gewaltenteilung}

Die bikamerale Gewaltenteilung beschreibt die Aufteilung der Staatsgewalt, in erster Linie der gesetzgebenden Gewalt, auf zwei Parlamentskammern. Sie steht also für die Gewaltenteilung innerhalb der Legislative. Die bikamerale Gewaltenteilung kann als Teil der horizontalen Gewaltenteilung oder sofern die zweite Kammer wie in Deutschland und Österreich eine Länderkammer bildet - als Teil der föderativen Gewaltenteilung (Steffani 1979: 26) betrachtet werden. Entscheidend ist jedoch, dass sie aufgrund der starken Dominanz der Parteipolitik faktisch verhindert wird, wenn beide Kammern von der gleichen parteipolitischen Mehrheit kontrolliert werden. Die Parteipolitik dominiert schließlich auch in Länderkammern wie dem deutschen und österreichischen Bundesrat, obwohl diese der Artikulation von Länderinteressen dienen sollten. Eine vergleichende Betrachtung Großer und Kleiner Koalitionen in beiden Ländern zeigt, dass die bikamerale Gewaltenteilung bei Großen Koalitionen im Gegensatz zu Kleinen Koalitionen in aller Regel faktisch nicht möglich bzw. relativ unwahrscheinlich ist.

Bei Kleinen Koalitionen ist die Gewaltenteilung innerhalb der Legislative faktisch möglich bzw. relativ wahrscheinlich, da die Wahlen auf Landesebene in der zweiten Kammer verhältnismäßig leicht andere politische Kräfteverhältnisse als in der ersten Kammer erzeugen können. In Deutschland und Österreich verfügten Kleine Koalitionen häufig über keine Mehrheit im Bundesrat. 
Bei Großen Koalitionen ist die Gewaltenteilung innerhalb der Legislative faktisch nicht möglich bzw. relativ unwahrscheinlich, da die Wahlen auf Landesebene in der zweiten Kammer nicht bzw. nur unter bestimmten Umständen andere politische Kräfteverhältnisse als in der ersten Kammer erzeugen können. So hatte in Österreich keine und in Deutschland bisher nur eine Große Koalition zum Teil keine Mehrheit im Bundesrat: die zweite Große Koalition nach der Bildung der CDU/FDP-Koalition in Hessen 2009.

Der Ländervergleich zeigt, dass die Mehrheiten Großer Koalitionen im deutschen Bundesrat kleiner und unbeständiger sind als im österreichischen. Dies ist auf die spezifischen Abstimmungsmodalitäten in der deutschen Länderkammer zurückzuführen: Beschlüsse können nur mit absoluter Mehrheit gefasst werden (Art. 52 Abs. 3 Satz 1 GG), die Stimmen eines Landes dürfen nur einheitlich abgegeben werden (Art. 51 Abs. 3 Satz 2 GG) und Enthaltungen bei zustimmungsbedürftigen Gesetzen entfalten die Wirkung von NeinStimmen. Daher ist es in Deutschland im Gegensatz zu Österreich faktisch möglich, dass eine Große Koalition keine Mehrheit im Bundesrat hat. Der bikameralen Gewaltenteilung kommt in Deutschland allerdings eine weitaus größere Bedeutung zu als in Österreich, da in Deutschland auch nach der ersten Stufe der Föderalismusreform ein relativ großer Anteil der Gesetze der Zustimmung des Bundesrats bedarf, während der österreichische Bundesrat nur in wenigen Ausnahmefällen ein absolutes Vetorecht besitzt (Art. 35 Abs. 4; Art. 44 Abs. 2; Art. 50 Abs. 1 Satz 2 B-VG) bzw. als Vetospieler fungiert. Allerdings kann seit 1988 ein Drittel der Mitglieder des österreichischen Bundesrats eine abstrakte Normenkontrolle beantragen (Art. 140 Abs. 1 Satz 2 B-VG) - damit jedoch nicht die Opposition zu einer Großen Koalition.

Die faktische Verhinderung der bikameralen Gewaltenteilung bei Großen Koalitionen ist einerseits negativ zu bewerten. Schließlich bedürfen Gesetze, die von der Zustimmung der zweiten Kammer abhängen und damit eine besondere Bedeutung implizieren, nur eines Konsenses zwischen den Regierungsparteien und keines übergreifenden bzw. breiteren Konsenses.

Andererseits ist die Auswirkung Großer Koalitionen auf die bikamerale Gewaltenteilung positiv zu bewerten. Schließlich erhöht sich durch die parteipolitische „Absorbierung“ der zweiten Kammer (Tsebelis 2002: 145) das Steuerungs- bzw. Reformpotenzial im Bereich der von der Zustimmung dieser Kammer abhängigen Gesetzgebung. Dabei ist hervorzuheben, dass der Bundesrat in Deutschland bei Kleinen Koalitionen in Zeiten unterschiedlicher Mehrheiten in Bundestag und Bundesrat häufig die Ursache eines „Reformstaus" bildet (Tabelle 3 ). 
Tabelle 3

Der deutsche Bundesrat als Vetospieler

\begin{tabular}{|c|c|c|c|}
\hline Wahlperiode & $\begin{array}{l}\text { Mehrheit } \\
\text { im } \\
\text { Bundesrat }\end{array}$ & $\begin{array}{c}\text { Gesamtzahl der } \\
\text { Anrufungen des } \\
\text { Vermittlungsaus- } \\
\text { schusses }\end{array}$ & $\begin{array}{l}\text { Nicht verkündete } \\
\text { Gesetze nach } \\
\text { erfolgter } \\
\text { Vermittlung }\end{array}$ \\
\hline $\begin{array}{c}\text { 04. Wahlperiode } \\
(1961-1965)\end{array}$ & $\begin{array}{l}\text { Regierung } \\
\text { (KK) }\end{array}$ & 39 & 2 \\
\hline $\begin{array}{c}\text { 07. Wahlperiode } \\
(1972-1976)\end{array}$ & Opposition & 104 & 7 \\
\hline $\begin{array}{c}\text { 10. Wahlperiode } \\
(1983-1987)\end{array}$ & $\begin{array}{l}\text { Regierung } \\
\text { (KK) }\end{array}$ & 6 & 0 \\
\hline $\begin{array}{c}\text { 13. Wahlperiode } \\
\text { (1994-1998) }\end{array}$ & Opposition & 92 & 10 \\
\hline $\begin{array}{c}\text { 15. Wahlperiode } \\
(2002-2005)\end{array}$ & Opposition & 102 & 12 \\
\hline $\begin{array}{c}\text { 16. Wahlperiode } \\
(2005-2009)\end{array}$ & $\begin{array}{l}\text { Regierung }^{\mathrm{a}} \\
\text { (GK) }\end{array}$ & 8 & $2^{b}$ \\
\hline
\end{tabular}

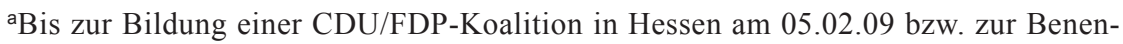
nung der Mitglieder durch die neu gewählte hessische Landesregierung am 09.02.09.

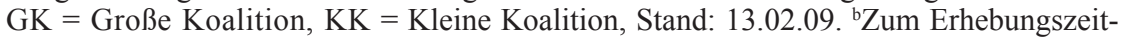
punkt noch im Vermittlungsverfahren. Quelle: Eigene Darstellung (Basis: Informationen des Bundesrats).

Bei Großen Koalitionen besteht in Deutschland in aller Regel - ähnlich wie bei Kleinen Koalitionen in Zeiten gleicher Mehrheiten in Bundestag und Bundesrat - kaum die Gefahr einer Entscheidungsblockade durch den Bundesrat (Tabelle 3). Dies bedeutet jedoch nicht zwingend, dass das erhöhte Steuerungs- bzw. Reformpotenzial konsequent genutzt wird. Einerseits ist eine erhöhte Reformtätigkeit wahrscheinlich, da alle großen Parteien in die Verantwortung eingebunden sind und keine davon als „Auffangbecken“ für Proteste gegen die Regierungspolitik fungieren kann. So ist v. a. zu Beginn bzw. in der ersten Hälfte der Wahlperiode sowie bei Großen Koalitionen, die aufgrund exogener Zwänge gebildet wurden, zu erwarten, dass wichtige und weitreichende Reformen umgesetzt werden. Andererseits ist eine erhöhte Reformtätigkeit unwahrscheinlich, da die beiden großen Parteien eine „Regierung der Rivalen“ (Hildebrand 1984: 241) bilden, sich ,gegeneinander und 
gegen die Regierung als Ganzes profilieren“ (Renzsch/Schieren 1997: 404) müssen und „,nur wenig Interesse am gemeinsamen Erfolg“ haben dürften (Renzsch/Schieren 1997: 404). Entscheidend ist, dass die Leistungsfähigkeit einer Regierung stark von der Kompromissfähigkeit der Regierungsparteien abhängt (Knorr 1975: 24) und diese bei Großen Koalitionen eher schwach ausgeprägt sein dürfte, da die großen Parteien gegeneinander konkurrieren müssen, um eine Kleine Koalition unter ihrer Führung bilden oder zumindest in einer Großen Koalition den Regierungschef stellen zu können (Müller 2008: 505 f.). So ist v. a. gegen Ende bzw. in der zweiten Hälfte der Wahlperiode sowie bei Großen Koalitionen, die (ausschließlich) aufgrund endogener Zwänge gebildet wurden, zu erwarten, dass überwiegend durch Minimalkompromisse regiert wird - und sich die Entscheidungsblockade, die bei Kleinen Koalitionen in Zeiten unterschiedlicher Mehrheiten in Bundestag und Bundesrat besteht, gewissermaßen vom Vermittlungsausschuss in den Koalitionsausschuss ,verlagert“.

\section{(3) Konstitutionelle Gewaltenteilung}

Die konstitutionelle Gewaltenteilung (Steffani 1979: 27) impliziert u. a. die Aufteilung der Gewalt zur Verfassungsänderung und Gesetzgebung. Zwar können die beiden Gewalten teilidentisch sein, jedoch muss die Gewalt zur Verfassungsänderung einen gewissen „Mehrwert“ aufweisen, da sonst das Prinzip der Normenhierarchie ,,ausgehöhlt“ werden würde. Die konstitutionelle Gewaltenteilung steht also für die Höherwertigkeit der Gewalt zur Verfassungsänderung gegenüber der Gewalt zur Gesetzgebung. Entscheidend ist, dass sie bei Verfassungsänderungen auf parlamentarischer Ebene faktisch verhindert bzw. eingeschränkt wird, wenn die Gewalt zur Verfassungsänderung in beiden bzw. einer der beiden Parlamentskammern von der gleichen parteipolitischen Mehrheit kontrolliert wird wie die Gewalt zur Gesetzgebung. Eine vergleichende Betrachtung Großer und Kleiner Koalitionen in beiden Ländern zeigt, dass die konstitutionelle Gewaltenteilung bei Großen Koalitionen im Gegensatz zu Kleinen Koalitionen in aller Regel faktisch nicht bzw. nur eingeschränkt gegeben ist (Tabelle 1 und 2).

Bei Kleinen Koalitionen ist die Höherwertigkeit der Gewalt zur Verfassungsänderung faktisch gegeben, da die Regierungsparteien nur über die gesetzgebende Gewalt verfügen. In Deutschland und Österreich gab es noch nie eine Kleine Koalition, die eine (garantierte) verfassungsändernde Mehrheit be$\mathrm{sa}$ - in Deutschland zwei Drittel der Mitglieder des Bundestags und des 
Bundesrats (Art. 79 Abs. 2 GG), in Österreich zwei Drittel der abgegebenen Stimmen bei einer Anwesenheit von mindestens der Hälfte der Mitglieder im Nationalrat (Art. 44 Abs. 1 B-VG) sowie auch im Bundesrat, sofern „die Zuständigkeit der Länder in Gesetzgebung oder Vollziehung eingeschränkt wird“" (Art. 44 Abs. 2 B-VG).

Bei Großen Koalitionen ist die Höherwertigkeit der Gewalt zur Verfassungsänderung in der ersten Parlamentskammer in aller Regel faktisch nicht gegeben, da die Regierungsparteien dort meist über beide Gewalten verfügen. So besaßen alle Großen Koalitionen in Deutschland und Österreich mit zwei Ausnahmen (den Regierungskoalitionen nach den Nationalratswahlen 1994 und 2008) eine (garantierte) verfassungsändernde Mehrheit im Bundestag bzw. Nationalrat. Der Ländervergleich zeigt jedoch, dass damit relativ große Unterschiede zwischen Deutschland und Österreich bestehen.

In Deutschland ist die konstitutionelle Gewaltenteilung bei Großen Koalitionen in aller Regel faktisch nur eingeschränkt gegeben, da diese meist über keine verfassungsändernde Mehrheit im Bundesrat verfügten, der allen Verfassungsänderungen zustimmen muss. Nur die zweite Große Koalition hatte über einen kleinen Zeitraum, von November 2006 bis Juli 2007, eine verfassungsändernde Mehrheit in beiden Kammern.

In Österreich ist die konstitutionelle Gewaltenteilung bei Großen Koalitionen in aller Regel faktisch nicht gegeben, da diese stets über eine verfassungsändernde Mehrheit im Bundesrat verfügten, der allerdings ohnehin nur bestimmten Verfassungsänderungen zustimmen muss. Außerdem hatte die Opposition meist nicht die notwendige Mehrheit, eine bei einer Teiländerung der Bundesverfassung mögliche Volksabstimmung zu verlangen. ${ }^{16}$ Von Bedeutung ist ferner, dass in Österreich ,[n] eben der eigentlichen Verfassung, dem Bundesverfassungsgesetz, [...] hunderte andere Verfassungsgesetze und Verfassungsbestimmungen in einfachen Gesetzen sowie eine Reihe weiterer ,Zwei-Drittel-Materien““ (Müller 2008: 510) existieren ${ }^{17}$ - und Große Koalitionen somit meist auch die Möglichkeit zur Regelung dieser Bereiche hatten.

16 Eine Gesamtänderung muss allerdings grundsätzlich einer Volksabstimmung unterzogen werden (Art. 44 Abs. 3 B-VG).

17 So kann z. B. das Bundesgesetz über die Geschäftsordnung des Nationalrats nur mit zwei Drittel der abgegebenen Stimmen bei einer Anwesenheit von mindestens der Hälfte der Mitglieder beschlossen werden (Art. 30 Abs. 2 Satz 2 B-VG). In Deutschland müssen indessen nur Abweichungen von den Vorschriften der Geschäftsordnung des Bundestags mit Zweidrittelmehrheit der anwesenden Mitglieder des Bundestags beschlossen werden $(\S 126 \mathrm{GOBT})$. 
Die faktische Verhinderung bzw. Einschränkung der konstitutionellen Gewaltenteilung bei Großen Koalitionen ist einerseits negativ zu bewerten. Schließlich bedürfen Verfassungsänderungen (in der ersten Parlamentskammer) nur eines Konsenses zwischen den Regierungsparteien und keines übergreifenden bzw. breiteren Konsenses. Dabei ist hervorzuheben, dass ein breiter Konsens im Bereich des Verfassungsrechts, der auch die Opposition zumindest zum Teil einschließt, aufgrund der Normenhierarchie bzw. der besonderen Bedeutung des Verfassungsrechts weitaus wichtiger ist als im Bereich des einfachen Rechts.

Andererseits ist die Auswirkung Großer Koalitionen auf die konstitutionelle Gewaltenteilung positiv zu bewerten. Schließlich erhöht sich dadurch das Steuerungs- bzw. Reformpotenzial im Verfassungsbereich. Besonders wichtig ist dies, wenn Verfassungsreformen aufgrund gravierender Veränderungen, Herausforderungen oder Fehlentwicklungen dringend notwendig sind und bei Kleinen Koalitionen primär aufgrund des unterschiedlichen „Rollenverhaltens“ der beiden großen Parteien scheitern würden. Fraglich ist allerdings wieder, ob das erhöhte Steuerungs- bzw. Reformpotenzial konsequent genutzt wird.

\section{(4) Temporale Gewaltenteilung}

Die temporale Gewaltenteilung (Steffani 1979: 23) beschreibt die Begrenzung politischer Amtszeiten durch regelmäßig wiederkehrende Wahlen mit einer realen Machtwechselchance. Sie steht also grundsätzlich für die Möglichkeit der Opposition, bei (kompetitiven) Wahlen zur Regierung zu werden. Entscheidend ist, dass diese faktisch nur dann gegeben ist, wenn die Opposition nicht nur theoretische Chancen hat, an die Regierung zu gelangen. Eine vergleichende Betrachtung Großer und Kleiner Koalitionen in beiden Ländern zeigt, dass die temporale Gewaltenteilung bei Großen Koalitionen im Gegensatz zu Kleinen Koalitionen faktisch nicht bzw. nur eingeschränkt gegeben ist (Tabelle 1 und 2).

Bei Kleinen Koalitionen ist die Machtwechselchance faktisch uneingeschränkt gegeben, da ein vollständiger Regierungswechsel tatsächlich möglich ist. Dies gilt grundsätzlich auch unter den Bedingungen eines Zweieinhalbparteiensystems. Schließlich ist dort faktisch die Möglichkeit gegeben, dass die Oppositionspartei allein oder mit einer neuen parlamentarischen Kraft eine absolute Mehrheit erringt. Allerdings erfolgte ein vollständiger Regierungswechsel in Deutschland nur nach der Bundestagswahl 1998, als 
die CDU/CSU-FDP-Koalition von einer SPD-B'90/Die Grünen-Koalition abgelöst wurde, und in Österreich nur nach der Nationalratswahl 1970, als die ÖVP-Mehrheitsregierung von einer SPÖ-Minderheitsregierung abgelöst wurde.

Bei Großen Koalitionen ist die Machtwechselchance faktisch nicht bzw. nur eingeschränkt gegeben. Bei einer Fortsetzung der Großen Koalition ist de facto überhaupt kein Regierungswechsel möglich. Denkbar ist in diesem Fall nur ein Wechsel zwischen dem „Senior-“" und dem „Juniorpartner“. Bei einer Beendigung der Großen Koalition ist de facto nur ein unvollständiger Regierungswechsel möglich. Schließlich können die Oppositionsparteien (als Neben- bzw. Interessenparteien) nicht zu einer (geschlossenen) alternativen Regierungsmehrheit ,anwachsen“ - und damit ihre Alternativfunktion nicht im vollen Umfang erfüllen.

Die faktische Verhinderung bzw. Einschränkung der temporalen Gewaltenteilung bei Großen Koalitionen ist grundsätzlich negativ zu bewerten. Schließlich wird ein ,politisches Machtkartell“ gebildet, das eine „echte Opposition", also eine wirkliche Regierungsalternative, verhindert, den Prozess der Wahl entwertet und dabei ein fundamentales Funktionsprinzip der Demokratie verletzt bzw. - in den Worten Werner Kaltefleiters (1967: 23) - das „Lebenselixier demokratischer Regierungsweise“ vernichtet. Prinzipiell lebt die repräsentative Demokratie „,vom Wettbewerb der Ideen und Konzepte, von der Überzeugung der Wähler, wirklich eine Wahl zu haben und mit ihrer Stimme über die Zukunft mit zu entscheiden“ (Köcher 2006). Sind sie dazu nicht in der Lage und sich der Tatsache bewusst, die Regierung de facto nicht abwählen zu können, kann dies „Ohnmachtsgefühle und Fatalismus“ (Köcher 2006) hervorrufen. Vor allem lange Phasen Großer Koalitionen, die nicht aufgrund exogener Zwänge (hoher Intensität) und damit ohne wesentliche „,inhaltliche Rechtfertigung" gebildet wurden, sind vor diesem Hintergrund nicht förderlich für die demokratische Kultur, u. U. sogar „Gift für die Demokratie" (Köcher 2006).

Als positive Folge lässt sich allenfalls die Verhinderung von umfassenden Politikrevisionen mit negativen Implikationen sehen (,adversary politics“; Finer 1975), die mit häufigen vollständigen Regierungswechseln möglicherweise einhergehen. Schließlich lassen unvollständige Regierungswechsel, die bei Großen Koalitionen faktisch maximal möglich sind, grundsätzlich „moderatere“ Veränderungen erwarten als vollständige, wenngleich ,adversary politics“ auch bei vollständigen Regierungswechseln in modernen Demokratien eher unwahrscheinlich sind (Strohmeier 2005: 218 ff.). 


\section{d) Zusammenfassung und Definition der Monopolisierenden Großen Koalition}

Sowohl in Deutschland als auch in Österreich führten Große Koalitionen im Vergleich zu Kleinen Koalitionen zu einem höheren Repräsentationsgrad der Wähler auf der parlamentarischen Entscheidungsebene bzw. der Regierungsebene und einer umfangreicheren Stärke der Regierungsmehrheit im Parlament. Dabei fällt auf, dass in beiden Ländern die jeweiligen Anteile Großer Koalitionen zum Teil wesentlich größer waren als bei Kleinen Koalitionen, jedoch im Laufe der Zeit stark abgenommen haben.

Sowohl in Deutschland als auch in Österreich führten Große Koalitionen im Vergleich zu Kleinen Koalitionen in aller Regel zu einer faktischen Einschränkung der horizontalen Gewaltenteilung (in zum Teil unterschiedlicher Weise), einer faktischen Verhinderung der bikameralen Gewaltenteilung, einer faktischen Verhinderung bzw. Einschränkung der konstitutionellen Gewaltenteilung und einer faktischen Verhinderung bzw. Einschränkung der temporalen Gewaltenteilung. Die größten Unterschiede zwischen Deutschland und Österreich zeigen sich im Bereich der bikameralen und konstitutionellen Gewaltenteilung.

Der bikameralen Gewaltenteilung kommt in Deutschland eine sehr viel größere Bedeutung zu als in Österreich, da der deutsche Bundesrat in weitaus mehr Fällen ein absolutes Vetorecht hat als der österreichische. Allerdings sind die Mehrheiten Großer Koalitionen im deutschen Bundesrat aufgrund der spezifischen Abstimmungsmodalitäten kleiner und unbeständiger als im österreichischen. So ist es in Deutschland im Gegensatz zu Österreich faktisch möglich, dass eine Große Koalition keine Mehrheit im Bundesrat hat.

Die konstitutionelle Gewaltenteilung ist bei Großen Koalitionen in Österreich in aller Regel faktisch nicht gegeben, da diese meist über die notwendige(n) verfassungsändernde(n) Mehrheiten verfügten. Damit hatten sie auch die Möglichkeit, eine Reihe weiterer „Zwei-Drittel-Materien“ zu regeln. In Deutschland ist die konstitutionelle Gewaltenteilung bei Großen Koalitionen hingegen in aller Regel faktisch nur eingeschränkt gegeben, da diese zwar über eine verfassungsändernde Mehrheit im Bundestag, meist aber über keine verfassungsändernde Mehrheit im Bundesrat verfügten. Zudem muss der deutsche Bundesrat im Gegensatz zum österreichischen allen Verfassungsänderungen zustimmen.

Trotz der bestehenden Unterschiede wird deutlich, dass die meisten Großen Koalitionen in Deutschland und Österreich einen spezifischen Wesensgehalt 
bzw. ein besonderes Wirkungspotenzial entfalten. Indem sie im Vergleich zu Kleinen Koalitionen faktisch nicht abgewählt werden können und substanzielle zusätzliche politische Entscheidungsmöglichkeiten gewinnen bzw. zentrale gewaltenteilende Mechanismen „unterlaufen“, „monopolisieren“ sie politische Macht. Dabei ist hervorzuheben, dass sie politische Macht in zweifacher Hinsicht monopolisieren: zum einen - als Einheit betrachtet - im Rahmen ihrer Stellung auf dem Wählermarkt als faktisch einzige Regierungsalternative und zum anderen im zentralen politischen Entscheidungssystem als Träger weitreichender politischer Entscheidungsmöglichkeiten. Vor diesem Hintergrund lassen sich Große Koalitionen in Deutschland und Österreich als Monopolisierende Große Koalitionen etikettieren - sofern sie in der ersten Kammer über eine Zweidrittelmehrheit verfügen. Schließlich bildet diese Mehrheit in beiden Ländern v. a. mit Blick auf die horizontale und konstitutionelle Gewaltenteilung eine „kritische Mehrheit“.

\section{Fazit: Die Monopolisierende Große Koalition - Definition eines Auslaufmodells?}

Die Nationalratswahl 2008 glich einem politischen Erdrutsch: Die Große Koalition ,stürzte“ von 69,7 auf 55,2 \% der Wählerstimmen und damit von $73,2 \%$ auf 59,0 \% der Parlamentsmandate. Damit ist die aktuelle Große Koalition in Österreich noch eine Große Koalition - allerdings keine Monopolisierende Große Koalition mehr. In Deutschland zeichnet sich in Umfragen ein ähnliches, wenn auch nicht ganz so „dramatisches“ Bild ab. Diese Entwicklung ist in beiden Ländern neben einer generellen Erosion großer Parteien bzw. Dekonzentration des Parteiensystems nicht zuletzt darauf zurückzuführen, dass die dritte Phase Großer Koalitionen (wie auch schon der letzte Abschnitt der zweiten Phase Großer Koalitionen) in Österreich und die zweite Große Koalition in Deutschland ausschließlich aufgrund endogener Zwänge, also ohne ,inhaltliche Rechtfertigung“, zustande kamen, was prinzipiell eine schlechte Voraussetzung für die Funktionsfähigkeit Großer Koalitionen und deren Akzeptanz durch die Wähler ist. Zudem finden Letztere in beiden Ländern mittlerweile ein relativ breites Spektrum an Oppositionsparteien bzw. parteipolitischen Alternativen vor, über das sie ihre Unzufriedenheit mit einer Großen Koalition zum Ausdruck bringen können.

Entscheidend ist, dass sich mit der Struktur des Parteiensystems auch der Charakter Großer Koalitionen ändert. Im Gegensatz zu einem von zwei Parteien dominierten Mehrparteiensystem führen „dekonzentriertere“ Mehrpar- 
teiensysteme - nach der Parteiensystemeinteilung von Ware (1996: 162) „even party systems“ - nicht zu Monopolisierenden Großen Koalitionen, unter Umständen sogar nur zu Großen Koalitionen als Minderheitskoalition. Auf jeden Fall könnte die in Deutschland und Österreich zu beobachtende Erosion der großen Parteien bzw. Dekonzentration des Parteiensystems dazu führen, dass der bei einer Gesamtbetrachtung bislang dominierende Typ Großer Koalitionen in beiden Ländern zu einem Auslaufmodell wird - jedoch nicht unbedingt die Große Koalition. Schließlich besteht ein wesentliches Problem darin, dass - wie die Nationalratswahl 2008 in Österreich zeigt - die Stimmenverluste der großen Parteien genau das Gegenteil von dem bewirken können, was sie wohl bewirken sollten: die Fortsetzung der Großen Koalition.

\section{Literatur}

Axelrod, Robert, 1970: Conflicts of Interest, Chicago.

Finer, Samuel E., 1975: Adversary Politics and Electoral Reform, London.

De Swaan, Abram, 1973: Coalition Theories and Coalition Formations, Amsterdam.

Gamson, William, 1961: A Theory of Coalition Formation, in: American Sociological Review 26 (3), 373-382.

Gassert, Philipp, 2008: Zweimal Große Koalitionen, in: Hans-Peter Schwarz (Koord.), Die Bundesrepublik Deutschland, München.

Hildebrand, Klaus, 1984, Von Erhard zur Großen Koalition 1963-1969, Stuttgart.

Infratest dimap, 2005: ARD DeutschlandTREND September 2005, http:// www.infratest-dimap.de (Stand: 18.03.07).

Infratest dimap, 2006: ARD DeutschlandTREND November 2006, http:// www.infratest-dimap.de (Stand: 20.11.08).

Jesse, Eckhard, 2008: „Extremistische Parteien“, in: APuZ, B 47, 7-11.

Kaltefleiter, Werner, 1967: Die Große Koalition, in: APuZ, B 18-19, 3-23.

Kielmansegg, Peter Graf, 2002: Mehrheiten sind nicht mehr garantiert, in: FAZ, 23.08.02, S. 9.

Kielmansegg, Peter Graf, 2007: Das geteilte Land, München.

Kiesinger, Kurt Georg, 1971: Regierungserklärung des Bundeskanzlers am

13. Dezember 1966 vor dem Deutschen Bundestag in Bonn, in: Hans

Ulrich Behn (Hrsg.), Die Regierungserklärungen der Bundesrepublik Deutschland, München, 185-204. 
Knorr, Heribert, 1975: Der parlamentarische Entscheidungsprozess während der Großen Koalition 1966 bis 1969, Meisenheim am Glan.

Köcher, Renate, 2006: Gift für die Demokratie, in: http://www.faz.net (Stand 19.10.06).

Kropp, Sabine/Schüttemeyer Suzanne S./Sturm, Roland, 2002: Koalitionen in West- und Osteuropa, in: dies. (Hrsg.), Koalitionen in West- und Osteuropa, Opladen, 7-41.

Kropp, Sabine, 2008: Koalitionsregierungen, in: Oscar W. Gabriel/dies. (Hrsg.), Die EU-Staaten im Vergleich, Wiesbaden, 515-549.

Lehmbruch, Gerhard, 1996: Die korporative Verhandlungsdemokratie in Westmitteleuropa, in: Swiss Political Science Review 2 (4), 1-41.

Leiserson, Michael A., 1966: Coalitions in Politics, Yale.

Müller, Wolfgang C., 1988: Die neue große Koalition in Österreich, in: Österreichische Zeitschrift für Politikwissenschaft 18 (4), 321-347.

Müller, Wolfgang C., 2008: Zur Leistungsfähigkeit großer Koalitionen, in: Zeitschrift für Staats- und Europawissenschaften 6 (3), 499-523.

Nolte, Detlef, 1988: Ist die Koalitionstheorie am Ende?, in: PVS 29 (2), 230-251. Pelinka, Peter, 1987: Der österreichische Weg: Wende oder Fortsetzung?, in:

Die Neue Gesellschaft/Frankfurter Hefte 34 (10), 926-934.

Pelinka, Anton, 1999: Demokratie: Österreich 1900 - Österreich 2000, in: Reinhold Gärtner (Hrsg.), Blitzlichter, Innsbruck/Wien, 9-25.

Pelinka, Anton, 2002: Koalitionen in Österreich: Keine westeuropäische Normalität, in: Sabine Kropp/Suzanne S. Schüttemeyer/Roland Sturm (Hrsg.), Koalitionen in West- und Osteuropa, Opladen, 69-87.

Pelinka, Anton/Rosenberger, Sieglinde, 2003: Österreichische Politik, Wien. Renzsch, Wolfgang/Schieren, Stefan, 1997: Große Koalition oder Minderheitsregierung, in: ZParl 28, 391-407.

Riker, William H., 1962: The Theory of Political Coalitions, New Haven.

Sartori, Giovanni, 1993: Parties and Party Systems, New York.

Schütt-Wetschky, Eberhard, 1990: Praxisorientierte Politikwissenschaft, in: Peter Haungs (Hrsg.), Wissenschaft, Theorie und Philosophie der Politik, Baden-Baden, 19-62.

Schuett-Wetschky, Eberhard, 2001: Gewaltenteilung zwischen Bundestag und Bundesregierung?, in: Klaus Dicke (Hrsg.), Der demokratische Verfassungsstaat in Deutschland, Baden-Baden, 67-117.

Schuett-Wetschky, Eberhard, 2005: Parlamentarismuskritik ohne Ende?, in: ZPol 15, 489-507.

Steffani, Winfried, 1979: Parlamentarische und präsidentielle Demokratie, Opladen. 
Strohmeier, Gerd, 2005: Vetospieler - Garanten des Gemeinwohls und Ursachen des Reformstaus, Baden-Baden.

Strøm, Kaare, 1990: Minority Government and Majority Rule, Cambridge.

Tsebelis, George, 2002: Veto Players, Princeton.

Ware, Alan, 1996: Political Parties and Party Systems, Oxford.

Wengst, Udo, 1985: Auftakt zur Ära Adenauer, Düsseldorf.

Korrespondenzanschrift:

Prof. Dr. Gerd Strohmeier

Technische Universität Chemnitz

Lehrstuhl für Europäische Regierungssysteme im Vergleich

Thüringer Weg 9

09126 Chemnitz

E-Mail: gerd.strohmeier@phil.tu-chemnitz.de

Web: http://www.tu-chemnitz.de/phil/politik/eur/

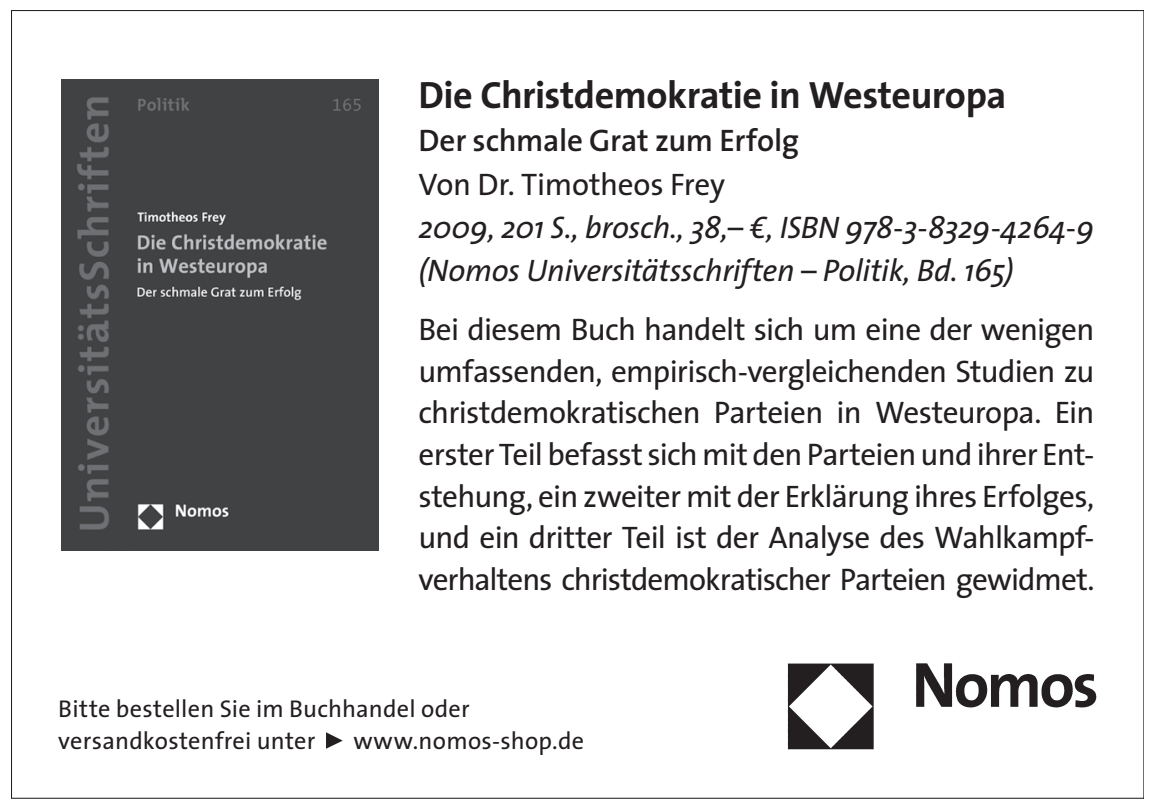

